\title{
Factors associated with alcohol use among minority female adolescents receiving preventive text messaging for underage drinking
}

\author{
Meenal Sawant ${ }^{*}$, MM Hospital ${ }^{1}$, EF Wagner ${ }^{1}$, SL Morris ${ }^{1}$, LM Siqueira ${ }^{2}$ \\ From INEBRIA 12th Congress, \\ Atlanda, GA, USA. 24-25 September 2015
}

\section{Background}

There is a high prevalence of alcohol use among adolescents, especially among Hispanic teens in middle adolescence. This emphasizes the need for developmentally appropriate and culturally sensitive underage drinking brief interventions. Texting (i.e. SMS) is extremely popular among U.S. teenagers, and its advantages as a brief intervention includes wide reach, low cost, easy standardization, automation of health message delivery, and the ability to include multiple recipients concurrently.

\section{Material and methods}

We conducted an RCT to examine the effectiveness of preventive text messaging for underage drinking among a sample of predominantly (77.0\%) Hispanic youth. Participants $(n=375)$ were recruited from a large, urban adolescent medicine setting. After completing the baseline intake survey, each participant was randomly assigned to either intervention (i.e. 2 times/week alcohol-related prevention SMS's for 16 weeks) or control (assessment-only) groups. Follow-up assessments were conducted for all participants at post-treatment and 1-month post-treatment. In the present study, we analyzed baseline data to examine the association between psychosocial risk factors and past month alcohol use.

\section{Results}

The sample included females between 12 and 18 years old $(\mathrm{M}=15.90, \mathrm{SD}=1.49) .42 \%$ reported lifetime alcohol use and $24 \%$ reported past month use. Regression analyses (MPLUS; version 5) revealed that past month alcohol use and future drinking intentions were significantly associated with (a) alcohol availability $(\beta=0.315$ and $\beta=0.115$ respectively), and (b) the CRAFFT (screening tool for substance use risks and consequences; $\beta=1.312, \beta=0.174$ respectively).

\section{Conclusions}

These findings highlight the importance of considering factors such as availability of substances and drinking intentions in the development of underage drinking brief interventions for minority populations.

\section{Acknowledgements}

Funding for this research was provided by the Ware Foundation.

\section{Authors' details}

${ }^{1}$ School of Social Work, Florida International University, Miami, USA.

${ }^{2}$ Adolescent Medicine Unit, Nicklaus Children's Hospital, Miami, USA.

Published: 24 September 2015

doi:10.1186/1940-0640-10-S2-P10

Cite this article as: Sawant et al:: Factors associated with alcohol use among minority female adolescents receiving preventive text messaging for underage drinking. Addiction Science \& Clinical Practice 2015 10(Suppl 2):P10. 